\title{
Detecting a small Kuiper Belt object using archival data of HST's Fine Guidance Sensor
}

\author{
Shay Zucker ${ }^{\star a}$, Hilke E. Schlichting ${ }^{b c}$, Eran O. Ofek ${ }^{b}$, Mike Wenz $^{d}$, Re'em Sari ${ }^{b e}$, \\ Avishay Gal-Yam ${ }^{f}$, Mario Liviog ${ }^{g}$ and Ed Nelan ${ }^{g}$ \\ ${ }^{a}$ Department of Geophysics and Planetary Sciences, Tel Aviv University, Tel Aviv 69978, Israel \\ ${ }^{b}$ Department of Astronomy, 249-17, California Institute of Technology, Pasadena, \\ California 91125, USA \\ ${ }^{c}$ CITA, University of Toronto, 60 St George Street, Ontario, M5S 3H8, Canada \\ ${ }^{d}$ Goddard Space Flight Center, 8800 Greenbelt Road, Greenbelt, Maryland 20771, USA \\ ${ }^{e}$ Racah Institute of Physics, Hebrew University, Jerusalem 91904, Israel \\ ${ }^{f}$ Faculty of Physics, Weizmann Institute of Science, POB 26, Rehovot 76100, Israel \\ ${ }^{g}$ Space Telescope Science Institute, 3700 San Martin Drive, Baltimore, Maryland 21218, USA \\ E-mail: shayz@post.tau.ac.il, hilke.schlichting@gmail.com, \\ eran@astro.caltech.edu, mwenz@hst.nasa.gov, sari@phys.huji.ac.il, \\ avishay.gal-yameweizmann.ac.il,mlivio@stsci.edu, nelan@stsci.edu
}

The Kuiper Belt is a remnant of the primordial Solar System. Measurements of its size distribution constrain its accretion and collisional history, and the importance of material strength of Kuiper elt objects. Small, sub-kilometer-sized, Kuiper Belt objects elude direct detection, but the signature of their occultations of background stars should be detectable. Such an occultation event lasts typically a fraction of a second, thus making it a classical high time-resolution observation. Here we report an analysis of archival data of HST's Fine Guidance Sensors (FGS), that reveals an occultation by such a small object. The detection introduces the FGS as a valuable HTRA instrument. We discuss the statistical aspects regarding the validation of the detection claim, and its physical implications.

High Time Resolution Astrophysics (HTRA) IV - The Era of Extremely Large Telescopes May 5 - 7, 2010

Agios Nikolaos, Crete Greece

\footnotetext{
* Speaker.
} 


\section{Introduction}

A small Kuiper Belt object (KBO) crossing the line of sight to a star will partially obscure the stellar light, an event which can be detected in the star's light curve. For visible light, the characteristic scale of diffraction effects, known as the Fresnel scale, is given by $(\lambda a / 2)^{1 / 2} \approx$ $1.3 \mathrm{~km}$, where $a \approx 40 \mathrm{AU}$ is the distance to the Kuiper Belt and $\lambda \approx 600 \mathrm{~nm}$ is the wavelength of our observations.

Diffraction effects will be apparent in the star's light curve as a result of occulting KBOs provided that both the star and the occulting object are smaller than the Fresnel scale[1,2]. Occultations by objects smaller than the Fresnel scale are in the Fraunhofer regime. In this regime the diffraction pattern is determined by the size of the KBO and its distance to the observer, the angular size of the star, the wavelength range of the observations and the impact parameter between the star and the KBO. The duration of the occultation is approximately given by the ratio of the Fresnel scale to the relative velocity perpendicular to the line of sight between the observer and the KBO. Because the relative velocity is usually dominated by Earth's velocity around the Sun, which is $30 \mathrm{~km} \mathrm{~s}^{-1}$, typical occultations only last a short time of the order of a tenth of a second.

Extensive ground-based efforts have been conducted to look for optical occultations[3-6]. So far, these visible searches have announced no detections in the region of the Kuiper Belt (30-60 AU), but one of these quests claims to have detected some events beyond $100 \mathrm{AU}$ and at about $15 \mathrm{AU}$ (Ref. [4]). Unfortunately, ground-based surveys may suffer from a high rate of false-positives owing to atmospheric scintillation, and lack the stability of space-based platforms. The ground-breaking idea to search for occultations in archival RXTE X-ray data resulted in several claimed occultation events[7]. Later, revised analysis of the X-ray data[8-11] concluded that the majority of the originally reported events were most probably due to instrumental dead-time effects. Thus, previous reports of optical and X-ray events remain dubious[5] and their inferred $\mathrm{KBO}$ abundance is inconsistent with the observed break in the KBO size distribution, which was obtained from direct detections of large KBOs[12 - 14]. Furthermore, they are also difficult to reconcile with theoretical expectations, which predict collisional evolution for KBOs smaller than a few kilometers in size $[15,16]$ and hence a lower KBO abundance than inferred from extrapolation from KBOs with radii $r>50 \mathrm{~km}$.

\section{The Fine Guidance Sensors Data set}

There are three Fine Guidance Sensors (FGS) on board of Hubble Space Telescope (HST). Each FGS consists of four photomultipliers (PMTs). Nominal HST operation uses two FGS for guiding, with each FGS observing its own guide star. The photon counts recorded by each FGS are therefore different, but global instrumental artefacts and observatory level transients will display in both FGS and can therefore be identified and removed.

For the past 14 years, the FGS have been collecting photometric measurements of stars with $40 \mathrm{~Hz}$ time resolution, allowing for the detection of a $\mathrm{KBO}$ occultation diffraction pattern rather than a simple decrease in the photon count.

Observations of the inclination distribution of large KBOs find that about $75 \%$ have an inclination angle $|i| \lesssim 20^{\circ}[17-19]$. We therefore divide the FGS observations into a low ecliptic latitude 
$\left(|b|<20^{\circ}\right)$ and a high ecliptic latitude $\left(|b|>20^{\circ}\right)$ sample. The high-ecliptic latitude observations provide an excellent control sample.

The FGS guide stars span a broad range of magnitudes and spectral types. The signal-to-noise ratio, $\mathrm{S} / \mathrm{N}$, in a $1 / 40 \mathrm{sec}$ data bin depends on the magnitude of the star. We derived the angular sizes of guide stars by fitting the 2MASS[21] JHK and USNO-B1 BR[22] photometry with a black-body spectrum. About $66 \%$ of the stars in our data set turned out to subtend angular sizes less than 0.5 of the Fresnel scale at a distance of $40 \mathrm{AU}$. The diffraction pattern that is produced by a sub-km sized KBO occulting an extended background star is smoothed over the finite stellar disk. This effect becomes clearly noticeable for stars that subtend sizes larger than about 0.5 of a Fresnel scales[1,2] and it reduces the detectability of occultation events around such stars.

\section{Detection Procedure}

Our detection algorithm performs a template search with theoretical light curves and uses a $\chi^{2}$ fitting procedure to identify occultation candidates. Our survey is most likely to detect KBO occultation events caused by objects that are $200-500 \mathrm{~m}$ in radius given the $\mathrm{S} / \mathrm{N}$ of our data and for a power-law index of the KBO size distribution, $q$, between 3 and 4.5. Occultation events in this size range are in the Fraunhofer regime. The theoretical light curves for our search algorithm are therefore calculated in the Fraunhofer regime. Our templates are calculated for various impact parameters assuming a point source background star and are integrated over the $400-700 \mathrm{~nm}$ wavelength range of the FGS observations. For a given impact parameter between the KBO and the star, our theoretical light curves have three free parameters that we fit for. The first is the mean number of photon counts, which is the normalization of the light curve. The second is the amplitude of the occultation, which is proportional to the size of the KBO, and the third is the width of the occultation, which is independent of the object size, and is determined by the ratio of the Fresnel scale to the relative speed between HST and the KBO perpendicular to the line of sight. This relative speed is determined by the combination of HST's velocity around Earth, Earth's velocity around the Sun and the velocity of the KBO itself. We use this information to restrict the parameter space for the template widths in our search such that we are sensitive to KBOs located at the distance of the Kuiper Belt between $30 \mathrm{AU}$ and $60 \mathrm{AU}$.

\section{Statistical Validation}

The significance of occultation candidates can be measured by their $\Delta \chi^{2}$ which is defined here as the difference between the $\chi^{2}$ calculated for the best fit of a flat light curve, which corresponds to no event, and the $\chi^{2}$ of the best fit template. Occultation events have large $\Delta \chi^{2}$, since they are poorly fit by a constant. Cosmic ray events, which give rise to one very large photon count reading in a $40 \mathrm{~Hz}$ interval, can also result in a large $\Delta \chi^{2}$ but the fit of the occultation template is very poor. We examined all flagged events for which the template fit of the diffraction pattern was better than $15 \sigma$. About a handful of false-positives where flagged by our detection algorithm that have a value of $\Delta \chi^{2}$ comparable to or larger than the occultation event we consider genuine. However, in all cases these false-positives were caused by a $1 \mathrm{~Hz}$ jitter due to the displacement of the guide star from its null position. To determine the $\Delta \chi^{2}$ detection criterion for our search 
algorithm and to estimate the probability that detected events are due to random noise we use the bootstrap technique[23]. Specifically, from a given FGS time series of length $N$ we randomly selected $N$ points with repetitions and created an 'artificial' time series from it. We analyzed these 'artificial' data sets using the same search algorithm that we applied to the actual FGS data. This technique creates random time series with noise properties identical to those of the actual data, but it will lose any correlated noise. Therefore, this technique is justified if there is no correlated noise in the data sets. To look for correlated noise we calculated the autocorrelation function, with lags between 0 to $1 \mathrm{sec}$. Most of the data sets are free of statistically significant correlated noise. The $\sim 12 \%$ of the data sets that did show correlated noise exceeding $4 \sigma$, which was often due to slopes (e.g., long-term variability) in the data sets, were excluded from the bootstrap analysis.

The ability to detect an occultation event of a given size KBO depends on the impact parameter of the KBO, the duration of the event, the angular size of the star and the $\mathrm{S} / \mathrm{N}$ ratio of the data. We determined the detection efficiency of our survey by recovering synthetic events that we planted into the observed photometric time series by multiplying the actual FGS data with theoretical light curves of $\mathrm{KBO}$ occultation events. The synthetic events correspond to $\mathrm{KBO}$ sizes ranging between $130 \mathrm{~m}<r<650 \mathrm{~m}$, they have impact parameters from 0 to 5.5 Fresnel scales and a relative velocity distribution that is identical to that of the actual FGS observations. To account for the finite angular sizes of the stars we generated light curve templates with stellar angular radii of 0.1, 0.2, 0.3, 0.4, 0.6, 0.8 and 1 Fresnel scales distributed according to the actual distribution of the FGS guide stars angular radii, assuming a KBO distance of $40 \mathrm{AU}$. The modified light curves with the synthetic events were analyzed using the same search algorithm that we used to analyze the FGS data. We normalize our detection efficency for a given size $\mathrm{KBO}, \eta(r)$, to 1 for an effective detection cross section with a radius of one Fresnel scale.

The detection efficiency of our survey is $\sim 0.05(\sim 0.6)$ for objects with $r=200 \mathrm{~m}(r=500 \mathrm{~m})$ located at $40 \mathrm{AU}$. Note that this value for the detection efficency already accounts for the angular radii distribution of the guide stars

\section{First Detection}

We applied our search procedure so far on four and a half years of archival FGS data, which contain about 12,000 star hours of low ecliptic latitude $\left(|b|<20^{\circ}\right)$ observations. This search has yielded so far one single detection, at ecliptic latitude $14^{\circ}$, (Fig. 1). The best-fit parameters yield a KBO size of $r=520 \pm 60 \mathrm{~m}$ and a distance of $45_{-4}^{+5} \mathrm{AU}$ where we assumed a circular KBO orbit and an inclination of $14^{\circ}$. We have already reported about this detection in a Nature paper[24].

Using our bootstrap simulations, we estimate a probability of $\sim 2 \%$ that such an event is caused by statistical fluctuations over the whole analyzed FGS data set. We note that for objects on circular orbits around the Sun two solutions can fit the duration of the event. However, the other solution is at a distance of $0.07 \mathrm{AU}$ from Earth, and is therefore unlikely. It is also unlikely that the occulting object was located in the asteroid belt, because the expected occultation rate from asteroids is about two orders of magnitude less than our implied rate. Furthermore, an asteroid would have to have an eccentricity of order unity to be able to explain the duration of the observed occultation event. 

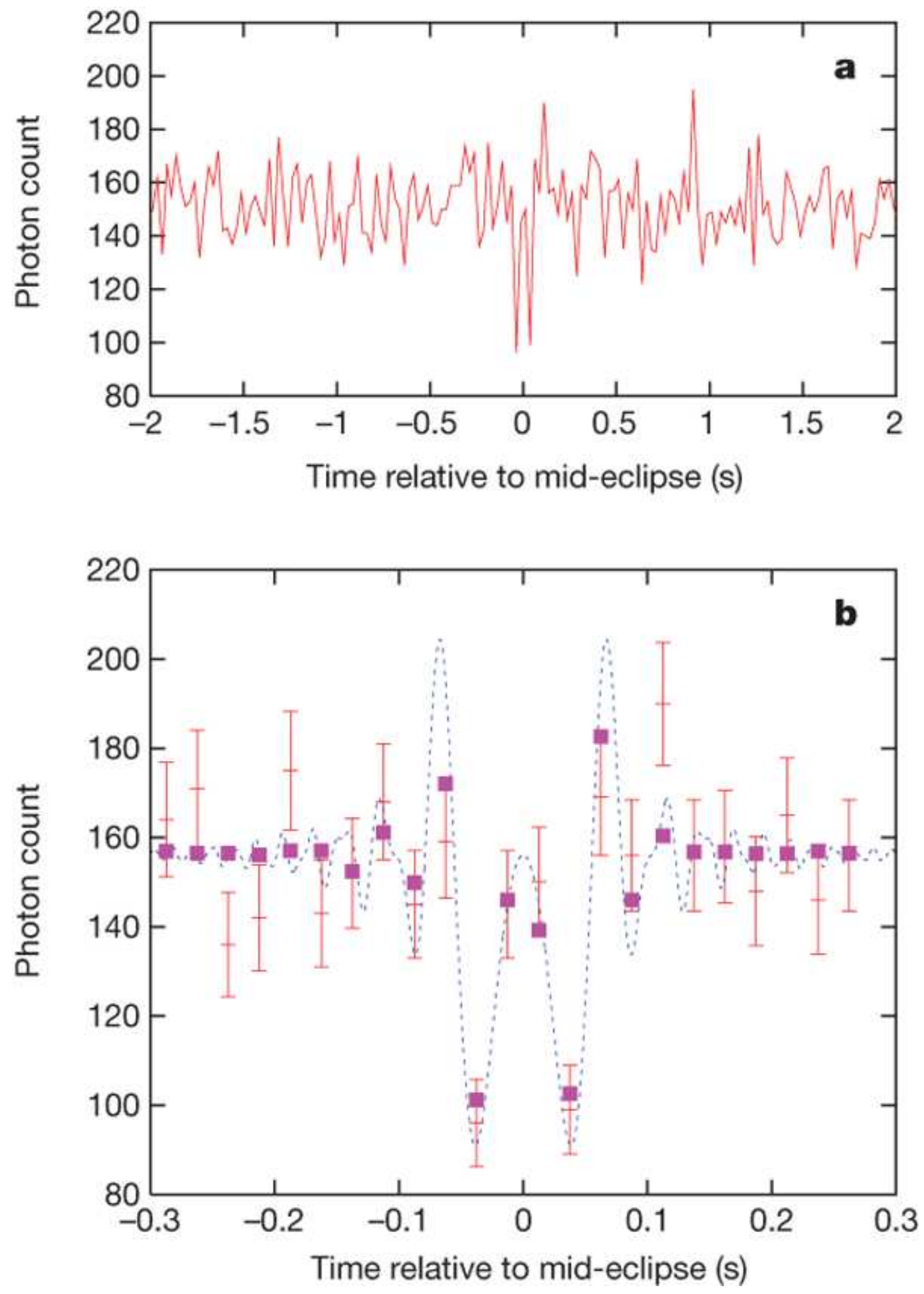

Figure 1: Photon counts as a function of time of the candidate occultation event observed by FGS2. (a) The photon count spanning 62 seconds around the occultation event. (b) The event in detail. The red crosses are the FGS data points with Poisson error bars, the dashed blue line is the theoretical diffraction pattern (calculated for the $400-700 \mathrm{~nm}$ wavelength range of the FGS observations), and the pink squares correspond to the theoretical light curve integrated over $40 \mathrm{~Hz}$ intervals. (Figure first published in [24]) 


\section{Physical Implications}

Using the KBO ecliptic latitude distribution from ref. [19], our detection efficiency, and our single detection, we constrain the surface density around the ecliptic (averaged over $-5^{\circ}<b<5^{\circ}$ of KBOs with radii larger than $250 \mathrm{~m}$ to $2.1_{-1.7}^{+4.8} \times 10^{7} \mathrm{deg}^{-2}$ [24]. This surface density is about three times the implied surface density at $5.5^{\circ}$ ecliptic latitude and about five times the surface density at $8^{\circ}-20^{\circ}$ ecliptic latitude. This is the first measurement of the surface density of hectometer-sized KBOs and it improves previous upper limits by more than an order of magnitude[3, 6].

Figure 2 displays our measurement for the sub-kilometer KBO surface density and summarizes published upper limits from various surveys. Our original data analysis focused on the detection of KBOs located at the distance of the Kuiper Belt between 30 and $60 \mathrm{AU}$. To compare our results with previously reported ground-based detections beyond 100AU[4], we performed a second search of the FGS data that was sensitive to objects located beyond the classical Kuiper Belt. Our results challenge the reported ground-based detections of two $300 \mathrm{~m}$-sized objects beyond $100 \mathrm{AU}$. Given our total number of star hours and a detection efficency of $3 \%$ for $300 \mathrm{~m}$-sized objects at $\sim 100 \mathrm{AU}$ we should have detected more than twenty occultations. We therefore rule out the previously claimed optical detections by more than $5 \sigma$. This result accounts for the broad latitude distribution of our observations (that is, $|b|<20^{\circ}$ ) and the quoted detection efficiency of our survey includes the effect of the finite angular radii of the guide stars at $100 \mathrm{AU}$.

The KBO cumulative size distribution is parameterized by $N(>r) \propto r^{1-q}$, where $N(>r)$ is the number of objects with radii greater than $r$, and $q$ is the power-law index. The power-law index for KBOs with radii above $\sim 45 \mathrm{~km}$ is $\sim 4.5$ (refs. [13, 16]) and there is evidence for a break in the size distribution at about $r_{\text {break }} \approx 45 \mathrm{~km}$ (refs. [12-14]). Hence we use this break radius and assume a surface density for KBOs larger than $r_{\text {break }}$ of $5.4 \mathrm{deg}^{-2}$ around the ecliptic[20]. Accounting for our detection efficiency, the velocity distribution of the HST observations, and assuming a single power-law for objects with radii less than $45 \mathrm{~km}$ in size, we find $q=3.9_{-0.3,-0.7}^{+0.3,+0.4}$ ( $1 \sigma$ and $2 \sigma$ errors) below the break[24]. Our results firmly show a deficit of subkilometer-sized KBOs compared to large objects. This confirms the existence of the previously reported break and establishes a shallower size distribution extending two orders of magnitude in size down to sub-kilometer-sized objects. This suggests that sub-kilometer-sized KBOs underwent collisional evolution, eroding the smaller KBOs. This collisional grinding in the Kuiper Belt provides the missing link between large KBOs and dust, producing debris disks around other stars. Currently, our results are consistent with a power-law index of strength-dominated collisional cascade[16], $q=3.5$, within $1.3 \sigma$ and with predictions for strengthless rubble piles[15], $q=3.0$, within $2.4 \sigma$. An intermediate value of $3<q<3.5$ implies that KBOs are strengthless rubble piles above some critical size, $r_{c}<r<45 \mathrm{~km}$, and strength dominated below it, $r<r_{c}$. Our observations constrain $r_{c}$ for the first time to our knowledge. At the $2 \sigma$ level we find $r_{c}>3 \mathrm{~km}$.

Using our estimate for the size distribution power-law index $(q=3.9)$ and our KBO surface density for $250 \mathrm{~m}$-sized KBOs at an ecliptic latitude of $b=5.5^{\circ}$, which is the ecliptic latitude of the RXTE observations of Scorpius X-1, we predict that there should be about $3.6 \times 10^{9}$ objects of radius $30 \mathrm{~m}$ per square degree. This is about 150 times less than the original estimate from $\mathrm{X}$ ray observations of Scorpius X-1 that reported 58 events[7], and it is about 30 times less than the revised estimate from the same X-ray observations, which concludes that up to 12 events might be 


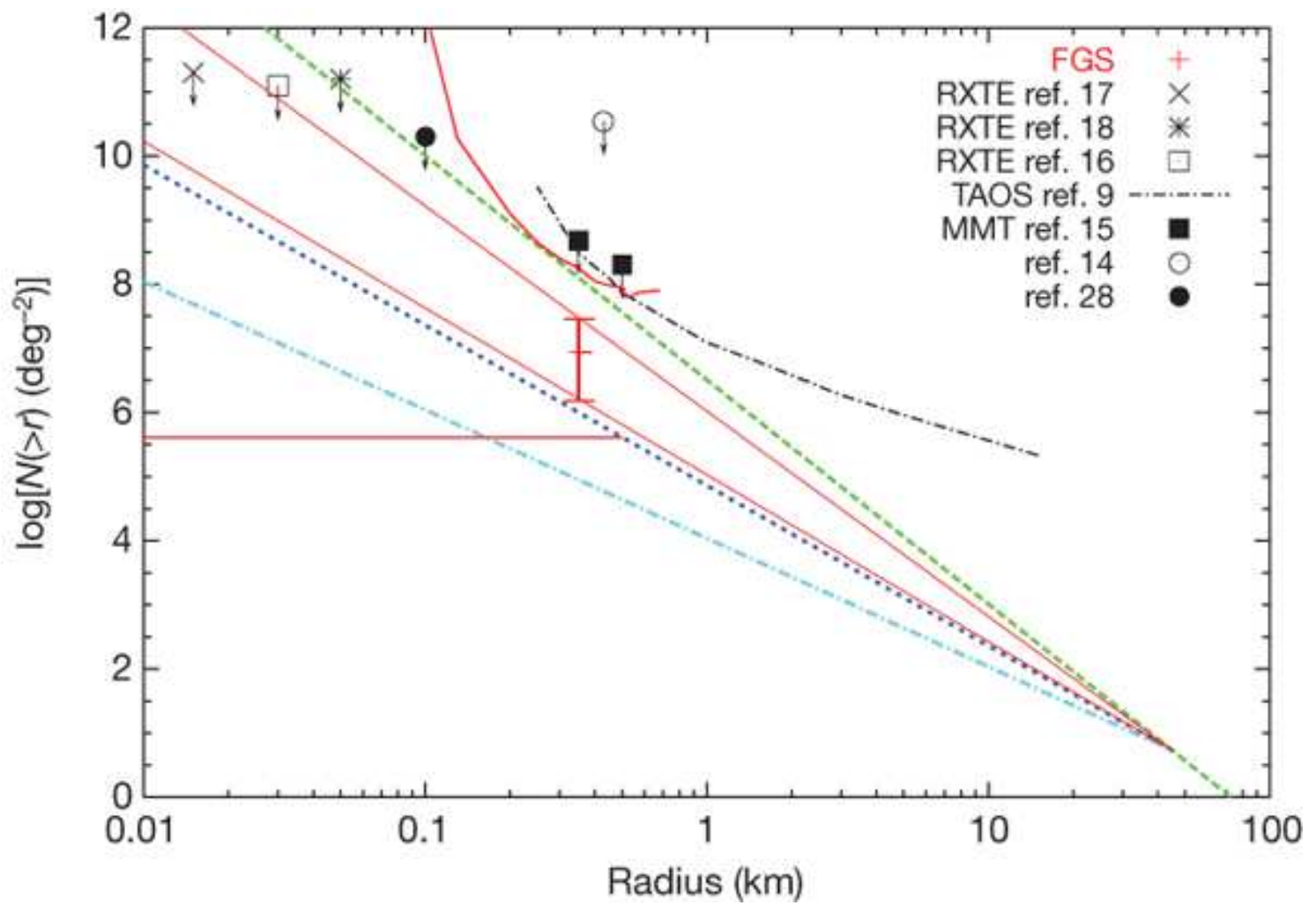

Figure 2: The results from our FGS survey are shown in red and are presented in three different ways. (1) The red cross is derived from our detection and represents the KBO surface density around the ecliptic (averaged over $-5^{\circ}<b<5^{\circ}$ ) and is shown with $1 \sigma$ error bars. The cross is plotted at $r=250 \mathrm{~m}$, which is roughly the peak of our detection probability (2) The upper and lower red curves correspond to our upper and lower 95\% confidence levels which were derived without assuming any size distribution. (3) The region bounded by the two straight red lines falls within $1 \sigma$ of our best estimate for the power-law size distribution index, that is, $q=3.9 \pm 0.3$, which was calculated for low ecliptic latitudes $\left(|b|<5^{\circ}\right)$. These lines are anchored to the observed surface density at $r=45 \mathrm{~km}$. For comparison, we also show three other lines (green, blue, turquoise). The green (long-dashed) line is the observed size distribution of large KBOs (that is, $r>45 \mathrm{~km}$ ), which has $q=4.5$, extrapolated as a single power-law to small sizes. The blue (short-dashed) line is a double power-law with $q=3.5$ (collisional cascade of strength-dominated bodies) for KBOs with radii less than $45 \mathrm{~km}$ and $q=4.5$ above. The turquoise (dot-dashed) line corresponds to $q=3.0$ (collisional cascade of strengthless rubble piles) for KBOs below $45 \mathrm{~km}$ in size. All distributions are normalized to $N(>r)=5.4 \mathrm{deg}^{-2}$ at a radius of $45 \mathrm{~km}$ (ref. [20]. In addition, 95\% upper limits from various surveys are shown in black (refs. [3, 5, 6, 8-10,25]. We note that a power-law index of 3.9 was used for calculating the cumulative KBO number density from the RXTE observations. (Figure first published in [24]) 
actual KBO occultations[8]. Our results rule out the implied surface density from these 12 events at $7 \sigma$ confidence level. One can reconcile our results and the claimed X-ray detections only by invoking a power-law index of $q \approx 5.5$ between $250 \mathrm{~m}$ and $30 \mathrm{~m}$. More recent X-ray work reports no new detections in the region of the Kuiper Belt but places an upper limit of $1.7 \times 10^{11} \mathrm{deg}^{-2}$ for objects of $50 \mathrm{~m}$ in radius and larger[10]. This is consistent with the KBO surface density of $N(>50 \mathrm{~m})=8.2 \times 10^{8} \mathrm{deg}^{-2}$ that we derive by extrapolating from our detection in the hectometer size range.

The statistical confidence level on our detection is $98 \%$. However, our conclusions that there is a significant break in the size distribution and that collisional erosion is taking place and the significant discrepancy with previously claimed occultation detections rely on the low number of events we discovered. These conclusions would only be strengthened if this event was caused by an unlikely statistical fluctuation or an as-yet-unknown instrumental artefact.

Ongoing analysis of the remaining FGS data, which will triple the number of star hours, together with further development of our detection algorithm (that is, including a larger number of light-curve templates) holds the promise of additional detections of occultation events and will allow us to constrain the power-law index of the KBO size distribution further.

\section{Concluding Remark}

Our detection of a small KBO using the archival data of the FGS demonstrates the huge potential of the FGS and probably similar future instruments. The combination of the high time resolution at the level of $40 \mathrm{~Hz}$ and the location on a space platform renders this instrument ideal for occultation studies. We hope that other KBO occultation surveys, as well as other optical HTRA studies, benefit from our experience with the FGS.

\section{References}

[1] F. Roques \& M. Moncuquet, A detection method for small Kuiper belt objects: the search for stellar occultations, Icarus 147, 530 (2000).

[2] T.C. Nihei et al., Detectability of occultations of stars by objects in the Kuiper belt and Oort cloud. AJ 134, 1596 (2007).

[3] Z.-W. Zhang et al., First results from the Taiwanese-American Occultation Survey (TAOS), ApJ, 685 , L157 (2008).

[4] F. Roques et al., Exploration of the Kuiper belt by high-precision photometric stellar occultations: first results, AJ, 132, 819 (2006).

[5] S.J. Bickerton, J.J. Kavelaars, \& D.L. Welch, A Search for sub-km Kuiper belt objects with the method of serendipitous stellar occultations, AJ, 135, 1039 (2008).

[6] F.B. Bianco et al., A Search for occultations of bright stars by small Kuiper belt objects using Megacam on the MMT, AJ, 138, 568 (2009).

[7] H.-K. Chang et al., Occultation of X-rays from Scorpius X-1 by small transneptunian objects, Nature 442, 660 (2006).

[8] H.-K. Chang, J.-S. Liang, C.-Y. Liu, \& S.-K. King, Millisecond dips in the RXTE/PCA light curve of Sco X-1 and trans-Neptunian object occultation, MNRAS, 378, 1287 (2007). 
[9] T.A. Jones, A.M. Levine, E.H. Morgan, \& S. Rappaport, Production of millisecond dips in Sco X-1 count rates by dead time effects, ApJ, 677, 1241 (2008).

[10] C.-Y. Liu, H.-K. Chang, J.-S. Liang, \& S.-K. King, Millisecond dip events in the 2007 RXTE/PCA data of Sco X-1 and the trans-Neptunian object size distribution, MNRAS, 388, L44 (2008).

[11] A.W. Blocker, P. Protopapas, \& C.R. Alcock, A Bayesian approach to the analysis of time symmetry in light curves: reconsidering Scorpius X-1 occultations, ApJ, 701, 1742 (2009).

[12] G.M. Bernstein et al., The size distribution of trans-neptunian bodies, AJ, 128, 1364 (2004).

[13] C.I. Fuentes \& M.J. Holman, A SUBARU archival search for faint trans-neptunian objects, AJ, 136, 83 (2008).

[14] W.C. Fraser et al., The Kuiper belt luminosity function from $m(R)=21$ to 26, Icarus 195, 827 (2008).

[15] M. Pan \& R. Sari, Shaping the Kuiper belt size distribution by shattering large but strengthless bodies, Icarus, 173, 342 (2005).

[16] J.W. Dohnanyi, Collisional models of asteroids and their debris, J. Geophys. Res., 74, 2531 (1969).

[17] D. Jewitt, J. Luu \& J. Chen, The Mauna Kea-Cerro-Tololo (MKCT) Kuiper Belt and Centaur Survey, AJ, 112, 1225 (1996).

[18] M.E. Brown, The Inclination Distribution of the Kuiper Belt, AJ, 121,2804 (2001)

[19] J.L. Elliot et al., The Deep Ecliptic Survey: a search for Kuiper belt objects and centaurs. II. Dynamical classification, the Kuiper belt plane, and the core population, AJ, 129, 1117 (2005).

[20] C.I. Fuentes, M.R. George, \& M.J. Holman, A Subaru pencil-beam search for $m(R) \sim 27$ trans-neptunian bodies, ApJ, 696, 91 (2009).

[21] M.F. Skrutskie et al., The Two Micron All Sky Survey (2MASS), AJ, 131, 1163 (2006).

[22] D.G. Monet et al., The USNO-B catalog, AJ, 125, 984 (2003).

[23] B. Efron, The Jackknife, the Bootstrap and other resampling plans, Society for Industrial Mathematics (1982).

[24] H.E. Schlichting et al., A single sub-kilometre Kuiper belt object from a stellar occultation in archival data, Nature, 462, 895 (2009)

[25] F. Roques, G. Georgevits, \& A. Doressoundiram, The Kuiper Belt Explored by Serendipitous Stellar Occultations in The Solar System Beyond Neptune eds. M.A. Barucci, H. Boehnhardt, D.P. Cruikshank \& A. Morbidelli (University of Arizona Press, 2008), p. 545 